\title{
S5.6
}

\section{DIAGNOSTICA MOLECOLARE MICROBIOLOGICA DELLE INFEZIONI ASSOCIATE A DISORDINI LINFOPROLIFERATIVI}

\section{Pistello M.}

\section{Dipartimento di Patologia Sperimentale, Biotecnologie \\ Mediche, Infettivologia ed Epidemiologia \\ Università degli Studi di Pisa}

La disponibilità di metodi molecolari per la rilevazione di acidi nucleici ha avuto un notevole impatto nel settore della diagnostica microbiologica. A fronte di questa realtà ormai consolidata, la diagnostica molecolare è un settore estremamente dinamico ed oggetto di intensa ricerca che si traduce nella continua introduzione di nuove tecnologie ed affinamento e miglioramento di prestazioni delle tecniche già esistenti. In questo ambito, sistemi molecolari quantitativi basati su tecnologia real-time, sequenziatori automatici di acidi nucleici ed apparecchiature per estrazione automatica giocano un ruolo di primaria importanza nel nel laboratorio di virologia molecolare. Oltre agli aspetti legati ad automazione ed integrazione della filiera "estrazione acidi nucleici - test analitico - risultato", questi sistemi presentano potenzialità ed applicazioni che rendono possibile diagnosi rapida ed accurata, stadiazione della malattia, management del paziente e studio dei meccanismi eziopatogenetici e di interazione agente patogeno-ospite.

Nella diagnosi virologica di malattie linfoproliferative l'impiego di metodi molecolari è di particolare importanza in quanto $i$ virus associati a manifestazioni tumorali sono in genere endemici e ad elevata prevalenza nella popolazione sana dove instaurano infezioni persistenti. Ruolo primario del virologo clinico è quindi l'identificazione dell'agente virale e, soprattutto, se il virus isolato è la causa del tumore o se il suo ritrovamento è un fatto incidentale. In questa presentazione verranno illustrati i metodi molecolari tradizionali e più innovativi applicati in virologia molecolare. Particolare enfasi verrà posta ai metodi molecolari per identificazione e quantizzazione degli agenti virali associati a malattie linfoproliferative e sull'importanza del dato di laboratorio virologico nella diagnosi di infezione, definizione dello stato di malattia e, non ultimo, nella formulazione e nella valutazione dell'efficacia del protocollo terapeutico. 